\title{
Resultados e complicações da entubação bicanalicular em dacriocistorrinostomia externa
}

\author{
Results and complications of bicanalicular intubation in external \\ dacryocystorhinostomy
}

\author{
Gisele Scholte de Souza Vieira ${ }^{1}$ \\ Maria Emília Xavier²
}

Trabalho realizado no Hospital do Servidor Público Estadual "Francisco Morato de Oliveira" - HSPE-FMO - São Paulo (SP) - Brasil.

${ }^{1}$ Médica Assistente, Hospital do Servidor Público Estadual "Francisco Morato de Oliveira" - HSPE-FMO São Paulo (SP) - Brasil.

${ }^{2}$ Doutora, Coordenadora da Residência em Oftalmologia do - HSPE-FMO - São Paulo (SP) - Brasil.

Endereço para correspondência: Gisele Scholte de Souza Vieira. Av. Nova Independência, 722 - São Paulo (SP) CEP 04570-001

E-mail: gisele.scholte@terra.com.br

Recebido para publicação em 11.08.2007

Última versão recebida em 09.02.2008

Aprovação em 24.04.2008

Nota Editorial: Depois de concluída a análise do artigo sob sigilo editorial e com a anuência do Dr. João Amaro Ferrari Silva sobre a divulgação de seu nome como revisor, agradecemos sua participação neste processo.

\section{RESUMO}

Objetivo: Analisar a taxa de sucesso e complicações em pacientes com obstrução lacrimal, submetidos à entubação bicanalicular na cirurgia de dacriocistorrinostomia externa(DCR-Ex). Métodos: Foram analisados os dados dos pacientes operados no Hospital do Servidor Público Estadual de São Paulo. A entubação lacrimal foi realizada com tubo de silicone, removido após oito semanas. O sucesso da cirurgia foi considerado nos casos de pacientes sem epífora ou secreção ocular no pós-operatório, com boa passagem de fluido para a narina ou orofaringe. As complicações relacionadas com o tubo de silicone foram agrupadas em uma tabela. Resultados: Os pacientes foram operados no período de abril de 2002 a julho de 2006, com tempo de seguimento médio de três meses. Do total de 65 olhos obteve-se uma taxa de sucesso de $89,2 \%$. Sete pacientes apresentaram epífora no pós-operatório, dos quais 5 foram reoperados. Em 7 olhos houve extrusão do silicone com menos de 15 dias de pós-operatório, e neste grupo 1 paciente necessitou de reoperação. Seis olhos apresentaram complicações com o silicone: prolapso do tubo (4 casos), formação de granuloma na cavidade nasal (1 caso) e aderência dos pontos lacrimais (1 caso). Conclusões: Este grupo de pacientes apresentou taxa elevada de sucesso $(89,2 \%)$ com a cirurgia de DCR-Ex associada à entubação bicanalicular. A entubação intra-operatória com tubo de silicone não é isenta de complicações.

Descritores: Dacriocistorinostomia/métodos; Obstrução dos ductos lacrimais/cirurgia; Intubação/métodos; Osteotomia; Dacriocistite/cirurgia; Elastômeros de silicone

\section{INTRODUÇ̃̃O}

As desordens do sistema lacrimal podem ser classificadas funcionalmente em alterações de excreção (drenagem), secreção ou da qualidade da lágrima. Causam sintomas semelhantes sendo que o excesso de lágrimas é o mais comum ${ }^{(1)}$.

Anatomicamente o sistema de drenagem lacrimal inicia-se nos pontos lacrimais, localizados na porção medial da borda palpebral ${ }^{(2)}$. As lágrimas se acumulam no canto medial formando o lago lacrimal. Elas entram pelo ponto e percorrem o sistema lacrimal até o meato inferior, localizado abaixo da concha inferior no nariz ${ }^{(3)}$

Através de uma cuidadosa anamnese, exame externo e à lâmpada de fenda, pode-se fundamentar a causa do excesso de lágrimas. O diagnóstico deve ser confirmado através do teste de retenção do corante e através da irrigação do sistema lacrimal ${ }^{(1)}$. 
A dacriocistorrinostomia externa (DCR-Ex) foi primeiramente descrita por Toti em 1904(2). Tem sua indicação em obstrução baixa da via lacrimal, ou seja, ao nível do ducto nasolacrimal. Em geral são obstruções adquiridas, uma vez que a obstrução lacrimal congênita geralmente se resolve através de massagem e/ou sondagem lacrimal. É realizada uma anastomose entre o saco lacrimal e a cavidade nasal através de uma abertura óssea, formando uma nova passagem para a lágrima $^{(2)}$.

Entre as causas de insucesso da DCR-Ex podemos citar: identificação incorreta do saco lacrimal, osteotomia inadequada, fechamento da osteotomia por fibrose, queda do retalho saco lacrimal-mucosa nasal, recesso de saco lacrimal e distúrbios na cavidade nasal ${ }^{(4-6)}$.

A entubação lacrimal temporária consiste na colocação de um tubo de silicone dentro da via lacrimal ${ }^{(2)}$, o qual será removido após um determinado período de tempo. Foi associada à DCR-Ex inicialmente nos casos em que obstrução dos canalículos lacrimais também estava presente. Os primeiros relatos datam de 1950 quando foram utilizados tubos de polietileno em entubação monocanalicular. No final dos anos 50, a entubação era bicanalicular ${ }^{(7)}$.

O objetivo da entubação lacrimal associada à dacriocistorrinostomia (DCR) seria manter a patência da anastomose cirúrgica e do óstio intranasal até que a epitelização se complete. Vários tipos de materiais foram utilizados para a entubação como fios de sutura e tubos de polietileno e poliuretano ${ }^{(6)}$.

O tubo de silicone foi introduzido por Gibbs em 1967 e, desde então, tem sido o material de escolha para este procedimento $^{(6)}$.

O silicone também pode ser utilizado nos casos de obstrução lacrimal congênita submetidos à sondagem lacrimal, porém a entubação é pouco indicada e restrita a alguns casos de obstruções recidivadas ou pacientes com idade acima de um ano $^{(2)}$.

Aqueles que optam pela entubação bicanalicular de rotina associada à DCR-Ex alegam que a presença do tubo de silicone em todo o trajeto previne o fechamento e a cicatrização da osteotomia ${ }^{(8)}$. Porém há um grupo contrário defendendo que a presença do tubo de silicone acarreta várias complicações, além de não ser relevante, pois a cirurgia de DCR-Ex apresenta índices elevados de sucesso sem a entubação ${ }^{(9)}$.

As complicações pós-operatórias relacionadas à presença do tubo de silicone são descritas em vários trabalhos. As mais citadas são: migração retrógrada e irritação corneana, formação de granuloma em qualquer ponto inclusive na osteotomia, aderência ou erosão do ponto lacrimal, migração nasal e trauma ao septo nasal (6,10-11).

Há controvérsias em relação a indicações, vantagens e desvantagens da entubação bicanalicular associada à técnica cirúrgica de DCR-Ex.

Neste estudo, procuramos mostrar a nossa experiência em pacientes operados através da técnica de DCR-Ex seguida de entubação bicanalicular, resultados e complicações.

\section{MÉTODOS}

Foram analisados os dados de pacientes submetidos à DCR no Hospital do Servidor Público Estadual de São Paulo. Os pacientes foram selecionados para a cirurgia no ambulatório de Plástica Ocular para onde foram encaminhados após exame oftalmológico completo, quando a queixa principal era excesso de lágrimas e/ou saída de secreção ocular.

Os pacientes foram submetidos aos seguintes exames das vias lacrimais: biomicroscopia, teste de desaparecimento do corante, expressão do saco lacrimal e teste de irrigação lacrimal. No exame de biomicroscopia à lâmpada de fenda avaliouse a presença de estenose ou ectopia dos pontos lacrimais, presença de entrópio ou ectrópio palpebrais, triquíase ou outra alteração que pudesse justificar a queixa de excesso de lágrimas. A seguir, o teste de desaparecimento do corante era feito através da instilação de uma gota de fluoresceína a $2 \%$ no fórnice inferior conjuntival de ambos os olhos. Examinou-se o menisco lacrimal através da luz de cobalto da lâmpada de fenda, após 5 minutos da instilação. A expressão do saco lacrimal foi realizada com a compressão da pele na região do ligamento cantal medial, observando-se a saída de secreção mucosa ou purulenta através dos pontos lacrimais. No teste de irrigação lacrimal, após a instilação de anestésico tópico na conjuntiva bulbar, uma sonda de Bowman era introduzida a partir dos canalículos lacrimais até o saco lacrimal. Quando os canalículos estavam pérvios, colocava-se uma cânula e injetava-se soro fisiológico através de um deles (em geral o inferior). Se houvesse refluxo do soro pelo outro canalículo, o paciente era encaminhado para fazer o exame de dacriocistografia. Com a presença de soro na garganta ou no nariz não se prosseguia com a investigação. Após o resultado da dacriocistografia, se comprovada a obstrução ao nível do ducto lacrimonasal, eram pedidos exames pré-operatórios e a cirurgia programada.

A técnica cirúrgica utilizada de rotina foi DCR-Ex com entubação bicanalicular no mesmo ato cirúrgico. Os pacientes em que não foi feita a entubação foram excluídos do estudo. O material utilizado para entubação foi o tubo de silicone isolado (Silastic ${ }^{\circledast}$ com diâmetros interno de $0,51 \mathrm{~mm}$ e externo de $0,94 \mathrm{~mm}$ ). Foi colocado nos canalículos através da sonda de Bowman. Fazem parte do estudo três pacientes submetidos a cirurgias prévias para desobstrução lacrimal sem sucesso. Os itens analisados foram: sexo, idade, olho operado, quadro clínico no pré-operatório, complicações com o tubo de silicone no pós-operatório e resolução da obstrução lacrimal avaliada através do teste de irrigação lacrimal no pós-operatório. Estes dados foram encontrados nos prontuários dos pacientes, sendo considerados casos de sucesso aqueles em que o paciente não mais apresentava queixas de epífora e/ou secreção ocular e nos quais o teste de irrigação apresentava passagem do soro para a narina ou para a orofaringe.

\section{Técnica cirúrgica}

Todos os pacientes foram submetidos à cirurgia sob anestesia geral. Com o paciente em decúbito dorsal, foi feita a 
colocação de cotonóides embebidos em xilocaína a $2 \%$ com vasoconstrictor na fossa nasal do lado a ser operado.

Após a injeção local de anestésico com vasoconstrictor, fez-se a incisão vertical na pele e no músculo orbicular, aproximadamente à média distância entre o canto medial e o dorso do nariz, a abertura do periósteo, o descolamento do saco lacrimal e a exposição da fossa lacrimal. Foi feita a fratura do osso na posição mais inferior da fossa lacrimal e a partir deste ponto removeu-se o osso. A osteotomia prolongou-se por aproximadamente $10 \times 10 \mathrm{~mm}$. Colocou-se a sonda de Bowman através do canalículo inferior chegando-se até o saco lacrimal. Confeccionou-se o retalho do saco lacrimal e depois o retalho da mucosa nasal. Foi feita a entubação do canalículo inferior e depois do superior, com o tubo de silicone acoplado à sonda de Bowman (Figura 1).

Os tubos foram passados para a cavidade nasal através da pinça baioneta, e fixados na parte interna da fossa nasal com fio nylon 5.0. Foi feita sutura do retalho saco lacrimal-mucosa nasal através de dois pontos com fio vicryl 6.0. Suturou-se o periósteo também com o fio vicryl 6.0 e em seguida a pele com fio nylon 6.0. Feito o curativo ocular utilizando-se uma pomada oftálmica de antibiótico. Utilizou-se antibioticoterapia sistêmica no pós-operatório por uma semana assim como uma associação de corticosteróide e antibiótico na forma de colírio por um mês, com diminuição progressiva da freqüência diária. Rinosoro foi utilizado de rotina por 15 dias. Os pontos na pele foram retirados após uma semana e o tubo de silicone foi removido após dois meses.

O teste de irrigação lacrimal foi realizado $24 \mathrm{~h}$ após a cirurgia. Repetia-se o teste após 7, 15 e 30 dias, dois e seis meses.



Figura 1 - Material para entubação canalicular

\section{RESULTADOS}

Foram analisadas 65 DCR-Exs com entubação bicanalicular com tubo de silicone, realizadas em 59 pacientes no período de abril/2002 a julho/2006. Todos os pacientes apresentavam obstrução do ducto nasolacrimal.

Do grupo total, 46 eram mulheres e 13 eram homens, com idades variando entre 6 e 86 anos (média de 64). Em 6 pacientes a cirurgia foi realizada em ambos os olhos (12,3\%), 32 cirurgias no olho esquerdo $(52,4 \%)$ e 21 no olho direito $(35,3 \%)$.

Entre as queixas relatadas pelos pacientes no pré-operatório as mais comuns foram: presença de secreção ocular em 24 casos (37\%), excesso de lágrimas em 20 casos (30,7\%) e excesso de lágrimas com secreção em 7 pacientes $(10,7 \%)$. Caracterizouse quadro de dacriocistite crônica em 10 pacientes $(15,4 \%)$ e dacriocistite aguda em 3 pacientes (4,6\%) (Figura 2). Em 1 paciente foi diagnosticado quadro de celulite orbitária. Os diagnósticos de dacriocistite crônica e aguda foram feitos baseados na história clínica e no exame físico.

Do total de 65 olhos, 58 (89,2\%) tiveram resolução da epífora no pós-operatório. Em 7 pacientes houve persistência dos sintomas após a cirurgia. Deste grupo, 5 foram reoperados e 2 aguardam o agendamento da cirurgia.

Entre as complicações com o fio de silicone encontramos prolapso na primeira semana de pós-operatório em 4 pacientes, sendo necessária a refixação no nariz, aderência dos pontos lacrimais com seis semanas de pós-operatório, no qual foi feita a exérese cirúrgica da aderência sem intercorrências (Figura 3), e formação de granuloma no óstio lacrimonasal em um paciente cada (Tabela 1). Houve extrusão precoce do fio de silicone, antes de 15 dias, em 7 pacientes.

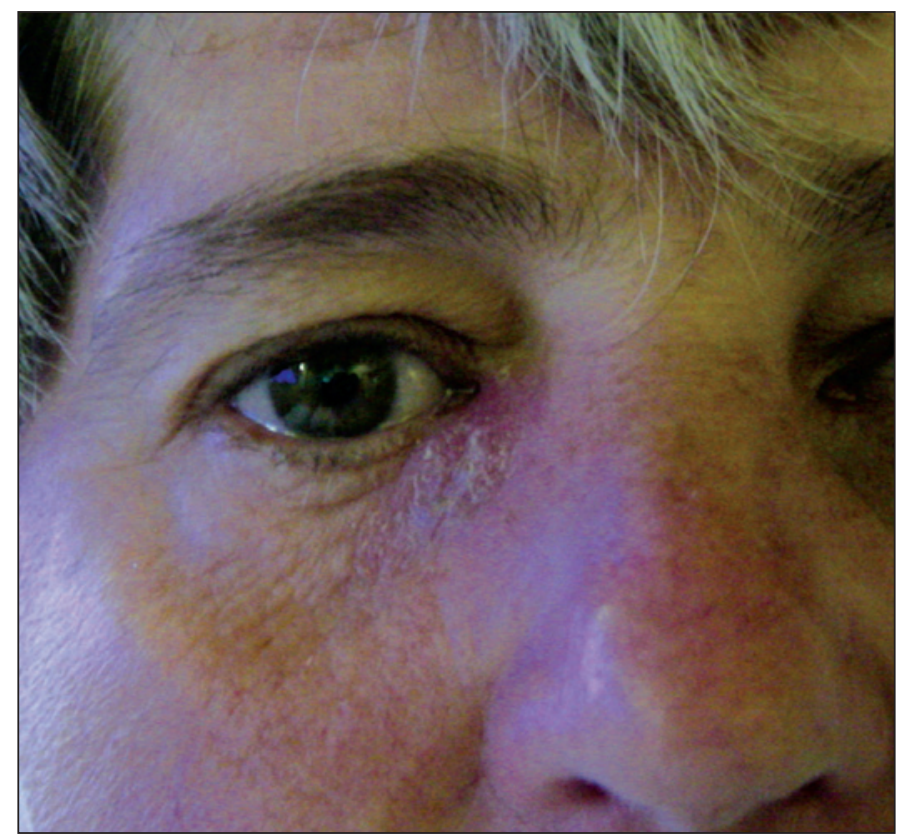

Figura 2 - Quadro de dacriocistite 




Figura 3 - Aderência dos pontos lacrimais

Tabela 1. Complicações com o tubo de silicone

\begin{tabular}{lc} 
Complicações & Número (\%) \\
Prolapso do tubo & $4(6,1 \%)$ \\
Aderência dos pontos lacrimais & $1(1,5 \%)$ \\
Formação de granuloma & $1(1,5 \%)$ \\
Total & $6(9,1 \%)$ \\
\hline
\end{tabular}

Não tivemos dificuldade na entubação intra-operatória em nenhum dos casos.

A passagem do soro fisiológico para a narina ou para a orofaringe no teste de irrigação feito no pós-operatório, foi interpretada como resolução da obstrução lacrimal. No grupo em que foi indicada a reoperação, o líquido injetado através de um dos canalículos refluiu pelo outro canalículo, caracterizando a persistência de uma obstrução lacrimal baixa, confirmada através da dacriocistografia.

\section{DISCUSS ÃO}

Houve uma predominância do sexo feminino neste estudo representando $78 \%$ dos casos operados, o que está de acordo com a literatura ${ }^{(12-13)}$. A média de idade foi de 64 anos, semelhante a outros estudos ${ }^{(14)}$.

As taxas de sucesso encontradas na literatura relacionadas à cirurgia de DCR são altas variando entre $80 \%$ e $98 \%$ dos casos operados ${ }^{(15-17)}$, semelhante ao resultado obtido neste grupo de pacientes. Apesar disto, muito se tem buscado para o aprimoramento da técnica, seja através de uma abordagem diferente como nas técnicas endoscópica ${ }^{(18-20)}$ ou transcana- licular ${ }^{(21)}$, pelo uso de substâncias no intra-operatório como a mitomicina $^{(22)}$ ou o 5 -fluouracil ${ }^{(23)}$, como também através da entubação lacrimal ${ }^{(6-7)}$.

Algumas indicações adotadas pela maioria dos cirurgiões para a entubação são: saco lacrimal atrófico, obstrução do canalículo comum detectada no pré-operatório, reoperação, retalhos do saco lacrimal-mucosa nasal inadequados e DCR por via endoscópica ${ }^{(24)}$.

Nós optamos por fazer a entubação lacrimal em todos os pacientes operados em nosso serviço por acharmos ser um procedimento simples, rápido e de custo baixo, associado ao menor risco de fechamento da nova passagem da lágrima; o tubo de silicone atuaria com uma barreira mecânica para que não houvesse a formação de fibrose na via lacrimal recémcriada $^{(6-7)}$.

As complicações encontradas em nossos pacientes listadas na tabela 1 apareceram em 9,1\% dos olhos, se considerarmos os casos de prolapso (4 olhos). É provável que o ponto no nariz para fixação do silicone estivesse colocado mais superficial do que o recomendável e que, inadvertidamente os pacientes os tenham soltado ao tocar a região. Como não houve comprometimento ocular em nenhum dos pacientes deste grupo, podemos não considerar como casos complicados pelo silicone. Assim a taxa de complicações diminuiria para $3 \%$, o que está compatível com a literatura ${ }^{(25)}$. Foi feito um reforço no ponto de fixação do silicone na fossa nasal, diminuindo assim o número de casos de prolapso e de extrusão precoce do silicone.

A aderência dos pontos lacrimais foi observada em 1 paciente no pós-operatório, sendo necessária uma intervenção cirúrgica que se fez sem intercorrências. Atribuímos esta complicação ao fato do silicone ter sido fixado muito apertado no conducto lacrimal. Recidiva da epífora ocorreu neste paciente sendo necessária a reoperação, porém não associamos o insucesso cirúrgico à complicação encontrada.

A formação de granuloma no local da osteotomia em 1 paciente foi verificada através de um exame endoscópico, sem prejuízo ao resultado final.

Nos 7 casos de insucesso, apenas o caso já citado apresentou complicação com o silicone no pós-operatório.

Em relação ao tempo de permanência do silicone, estudos mostram que é muito variado, permanecendo de uma semana a até seis meses ${ }^{(16)}$. Nos nossos pacientes optamos por deixar o silicone por dois meses por considerarmos um período adequado para a cicatrização dos tecidos presentes no óstio cirúrgico.

Não podemos afirmar que a entubação lacrimal vá aumentar a porcentagem de êxito cirúrgico. Para melhor avaliarmos esta porcentagem teríamos que analisar o fator cirúrgico, fácil ou difícil, volume do saco lacrimal, hemorragia durante o ato ou no pós-operatório, técnicas para realização das suturas e confecção dos retalhos.

Achamos serem necessários outros estudos para podermos comparar as taxas de sucesso da cirurgia de DCR-Ex com e sem entubação lacrimal. 


\section{CONCLUSÕES}

A taxa de sucesso da DCR-Ex encontrada neste estudo foi de $89,2 \%$. A entubação bicanalicular com o tubo de silicone é um procedimento simples, seguro e de fácil execução, embora não isento de complicações.

\section{ABSTRACT}

Purpose: To analyze the success rate and complications in patients with lacrimal obstruction submitted to surgeries. Methods: The data of patients operated in the "Hospital do Servidor Público Estadual of São Paulo" were analyzed. The lacrimal intubation was performed with a silicone tube, removed after 8 weeks. Surgeries were considered successful in those cases where patients had no postoperative epiphora or ocular secretion, and with a good passage of fluid to the nose or oropharynx. The complications related to the silicone tube were grouped in a table. Results: The patients were operated from April 2002 to July 2006 with an average follow-up period of three months. In a total of 65 eyes, success was obtained in $89.2 \%$ of the cases. Seven patients presented postoperative epiphora, and 5 of them were reoperated. In 7 eyes there was an extrusion of the silicone in less than 15 days from surgery, and in this group one patient needed a reoperation. Six eyes presented complications with the silicone: tube prolapse (4 cases), granuloma formation in the nasal cavity (1 case) and lacrimal puncta adhesion ( 1 case). Conclusions: This group of patients presented a high success rate with Ex-DCR surgery associated with lacrimal intubation. The intraoperative intubation with silicone tube is not free of complications.

Keywords: Dacryocystorhinostomy/methods; Lacrimal duct obstruction/surgery; Intubation/methods; Osteotomy; Dacryocystitis/surgery; Silicone elastomers

\section{REFERÊNCIAS}

1. Hornblass A, editor. Oculoplastic, orbital, and reconstructive surgery. Baltimore: Williams \& Wilkins; c1988.

2. Soares EJC, Moura EM, Gonçalves JOR, editores. Cirurgia plástica ocular. São Paulo: Roca; 1997.

3. Tse DT, editor. Color atlas of ophthalmic surgery: oculoplastic surgery. Philadelphia: J.B. Lippincott; 1992.
4. Diniz CM, Dantas RRA, Alvim HS, Rodrigues Júnior A, Figueiredo ARP Análise da evolução de pacientes com obstrução baixa de vias lacrimais submetidos à cirurgia de dacriocistorrinostomia externa. Rev Bras Oftalmol. 2003;62(6):414-9.

5. Dantas RRA. Dacriocistorrinostomia: sucesso x insucesso. Rev Bras Oftalmol. 1997;56(1):49-53.

6. Sodhi PK, Pandey RM, Malik KP. Experience with bicanalicular intubation of the lacrimal drainage apparatus combined with conventional external dacryocystorhinostomy. J Craniomaxillofac Surg. 2003;31(3):187-90.

7. Keith CG. Intubation of the lacrimal passages. Am J Ophthalmol. 1968; 65(1):70-4.

8. Bartley GB. Simultaneous silicone intubation through the osteotomy and the nasolacrimal duct during dacryocystorhinostomy. Am J Ophthalmol. 1996; 121(5):586-7.

9. Yigit O, Uzun H, Han T, Cakir BO. [The effect of bicanalicular silicone tube implantation on the success rate of endoscopic dacryocystorhinostomy]. Kulak Burun Bogaz Ihtis Derg. 2005;14(3-4):79-82. Turkish.

10. Brookes JL, Olver JM. Endoscopic endonasal management of prolapsed silicone tubes after dacryocystorhinostomy. Ophthalmology. 1999;106(11):2101-5.

11. Veloudios A, Harvey JT, Philippon M. Long-term placement of silastic nasolacrimal tubes. Ophthalmic Surg. 1991;22(4):225-7.

12. Schellini SA, Sakamoto RH, Samahá JT, Arangon F, Padovani CR. Dacriocistorrinostomia externa em hospital universitário: avaliação dos resultados. Arq Bras Oftalmol. 2004;67(2):301-4.

13. Mendes IR, Grumann Júnior A, Stefani E, Vegini F, Oechsler RA. Análise das dacriocistorrinostomias endonasais realizadas no Hospital Regional de São José - Homero de Miranda Gomes no período de 1999 a 2001. Rev Bras Oftalmol. 2006;65(3):152-6.

14. Sadiq SA, Ohrlich S, Jones NS, Downes RN. Endonasal laser dacryocystorhinostomy - medium term results. Br J Ophthalmol. 1997;81(12):1089-92. Comment in: Br J Ophthalmol. 1998;82(8):976.

15. Tarbet KJ, Custer PL. External dacryocystorhinostomy. Surgical success, patient satisfaction and economic cost. Ophthalmology. 1995;102(7):1065-70. Comment in: Ophthalmology. 1996;103(2):200.

16. Boboridis KG, Bruce C, Rose GE. Outcome of external dacryocystorhinostomy combined with membranectomy of a distal canalicular obstruction. Am J Ophthalmol. 2005;139(6):1051-5.

17. Hurwitz JJ, Merkur S, De Angelis D. Outcome of lacrimal surgery in older patients. Can J Ophthalmol. 2000;35(1):18-22.

18. Tsirbas A, Wormald PJ. Endonasal dacryocystorhinostomy with mucosal flaps Am J Ophthalmol. 2003;135(1):76-83.

19. Apaydin KC, Fisenk F, Karayalcin B, Akar Y, Saka O. Endoscopic transnasal dacryocystorhinostomy and bicanalicular silicone tube intubation. Ophthalmologica. 2004;218(5):306-11.

20. Knijnik D, Uebel VN, Santos RS. Dacriocistorrinostomia endoscópica endonasal em casos de epífora com vias lacrimais pérvias. Arq Bras Oftalmol 2003;66(2):173-5.

21. Kanecadan RT, Pinto LMAM, Nascimento EV, Werner PP, Vargas AU. Dacriocistorrinostomia transcanalicular com laser diodo: resultados preliminares. Arq Bras Oftalmol. 2006;69(5):691-4.

22. Liu D, Bosley TM. Silicone nasolacrimal intubation with mitomycin-C: a prospective, randomized, double-masked study. Ophthalmology. 2003;110(2):306-10.

23. Costa MN, Marcondes AM, Sakano E, Kara-José N. Endoscopic study of the intranasal ostium in external dacryocystorhinostomy postoperative. Influence of saline solution and 5-fluorouracil. Clinics. 2007;62(1):41-6.

24. Moura EM, Volpini M, Ianase M. Dacriocistorrinostomia transnasal vídeo-endoscópica com Nd:YAG laser e diodo laser. Arq Bras Oftalmol. 2004;67(5):807-14

25. Wang L, Chen D, Wang Z. New technique for lacrimal system intubation. Am J Ophthalmol. 2006;142(2):252-8. 